\title{
Inelastic and Elastic Mean Free Paths from FIB Samples of Metallic Glasses
}

\author{
D.T. Schweiss, Jinwoo Hwang, P.M. Voyles
}

Materials Science and Engineering, University of Wisconsin at Madison, Wisconsin 53706-1595, USA

In a TEM and STEM, knowledge of the sample thickness is crucial. Convergent beam electron diffraction and position averaged CBED can determine the sample thickness with very high accuracy but require single crystal samples. For small grain and amorphous samples, such as metallic glasses, other methods must be used.

We have used FIB and SEM to prepare samples of known thickness for measurement of the electron scattering elastic and inelastic mean free paths (EMFP and IMFP respectively) of three metallic glasses, $\mathrm{Cu}_{64.5} \mathrm{Zr}_{35.5}(\mathrm{CZ}), \mathrm{Zr}_{50} \mathrm{Cu}_{45} \mathrm{Al}_{5}$ (ZCA), and $\mathrm{Al}_{87} \mathrm{Y}_{7} \mathrm{Fe}_{5} \mathrm{Cu}_{1}$ (AYFC). Depositing carbon sidewalls on the FIBed lamellae dramatically improved the accuracy of the SEM thickness measurements, as shown in Fig. 1. We believe the carbon suppressed electron emission out the sides of the thin lamellae.

The EMFP and IMFP were measured using the log-ratio technique, in which $t / \lambda=\ln \left(I_{t} / I_{0}\right)[1,2]$, where $I_{t}$ is the total number of electrons incident on the sample, $I_{0}$ is the number of unscattered electrons, $\lambda$ is the mean free path, and $t$ is the thickness. We measured elastic scattering using a diffraction pattern. $I_{0}$ was the sum of the intensity in a 20 pixel by 20 pixel box around the zero beam. We measured inelastic scattering by EELS and the "compute thickness" function in DigitalMicrograph. Measurements were performed at $200 \mathrm{kV}$ with a convergence half angle of 0.74 mrad and an EELS collection half angle of $25 \mathrm{mrad}$.

The lamellae tended to warp in the FIB, so we adopted a wedge-shaped geometry, shown in Fig. 2(a). The thickness was measured at the thinnest and thickest points of each wedge, labeled I-IV in Fig. 2. The distance of the measurement point back from the trailing edge of the lamella was fixed using holes milled through the lamella with the FIB as fiduciaries.

Fig. 3 shows SEM thickness measurements against thickness in units of mean free path $(t / \lambda)$. The slope of these plots are the mean free path. At $200 \mathrm{kV}$, we find IMFP / EMFP values of $157 \pm 12$ / $43 \pm 4 \mathrm{~nm}, 158 \pm 5 / 38 \pm 1 \mathrm{~nm}$, and $135 \pm 5 / 88 \pm 1 \mathrm{~nm}$ for CZ, ZCA, and AYFC, respectively. The IMFP values disagree with available models by as much as $50 \%$. With known values of IMFP and EMFP, sample thickness can be very quickly determined for metallic glasses and other amorphous materials by quickly taking a diffraction pattern or EEL spectrum.

References

[1] D.C. Joy et al., SEM Inc., AMF, O'Hare, IL. (1979) pp. 817-826.

[2] R.D. Leapman, J. Microsc. (1984)239-253.

[3] This work was supported by NSF contract DMR-605890. Some of the FIB was done at the Electron Microscopy Center for Materials Research at Argonne National Laboratory, operated under Contract No. DE-AC02-06CH11357 by UChicago Argonne, LLC. 

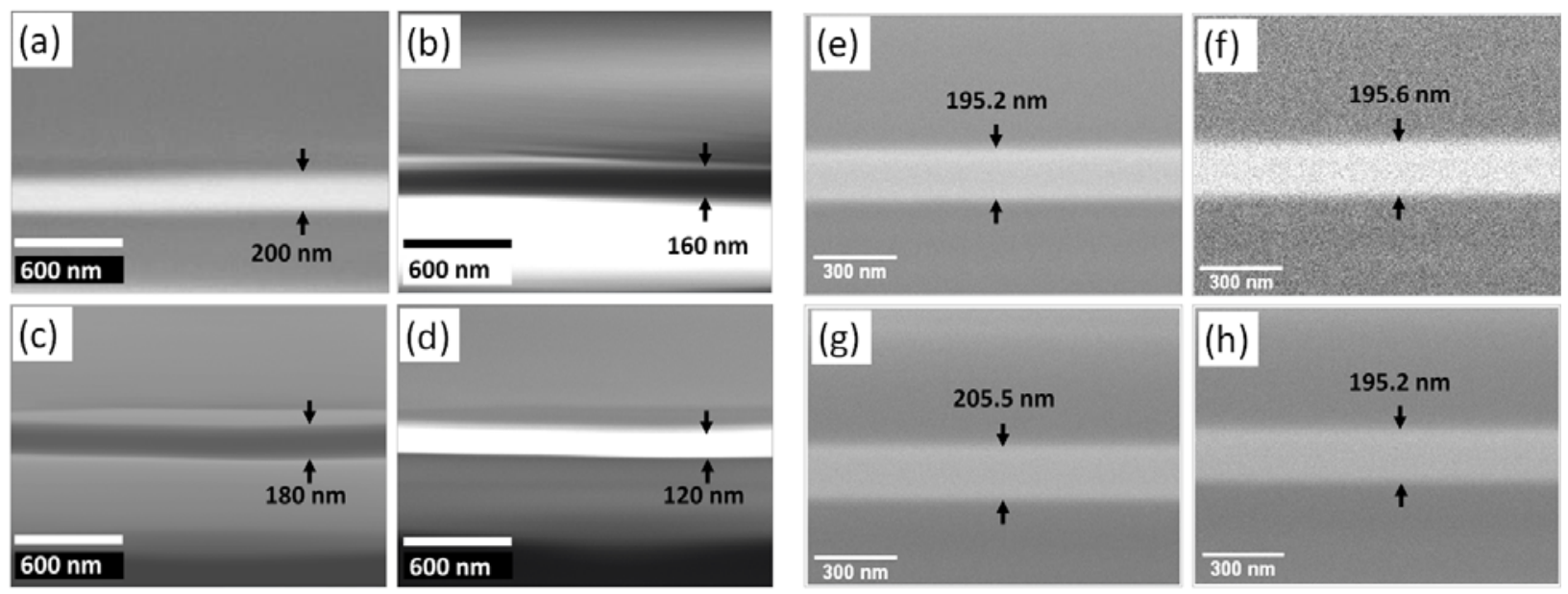

FIG. 1. (a)-(d) SEM images with carbon sidewalls using different detectors and accelerating voltages reveal large discrepancies in thickness measurements: (a) in-lens $\mathrm{SE}$ at $0.5 \mathrm{kV}$, (b) inlens SE at $20 \mathrm{kV}$, (c) in-lens SE at $3 \mathrm{kV}$, and (d) backscattered at $3 \mathrm{kV}$. (e)-(h) SEM images with carbon sidewalls (e) Everhart-Thornley (ET) at $3 \mathrm{kV}$, (f) backscatter at $3 \mathrm{kV},(\mathrm{g}) \mathrm{ET}$ at $7 \mathrm{kV}$, and (h) ET detector at $7 \mathrm{kV}$. For (a)-(d) the working distance (WD) $=2.4 \mathrm{~mm}$. For (e)-(g) WD = $11.7 \mathrm{~mm}$ and for $(\mathrm{h}) \mathrm{WD}=4.8 \mathrm{~mm}$.

(a)

(b)

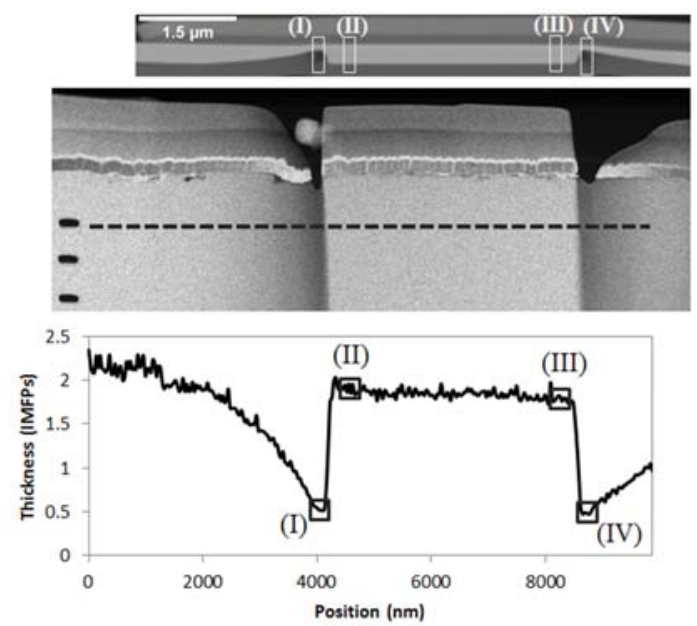

(d)

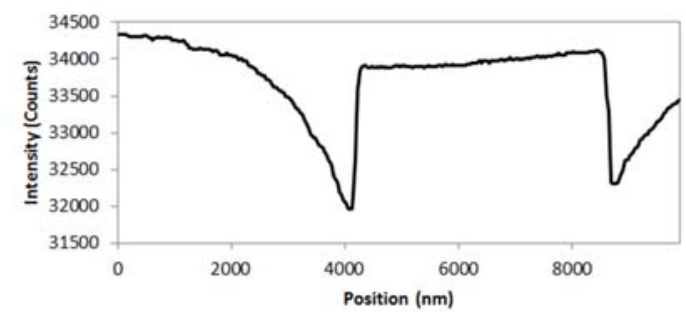

FIG. 2 (a) SEM image of lamella, (b) STEM image of lamella, (c) plot of thickness in units of IMFP, and (d) HAADF detector intensity. Dashed line in (b) is profile scan line. (I)-(IV) Indicate features seen in all scans which are at the same position.
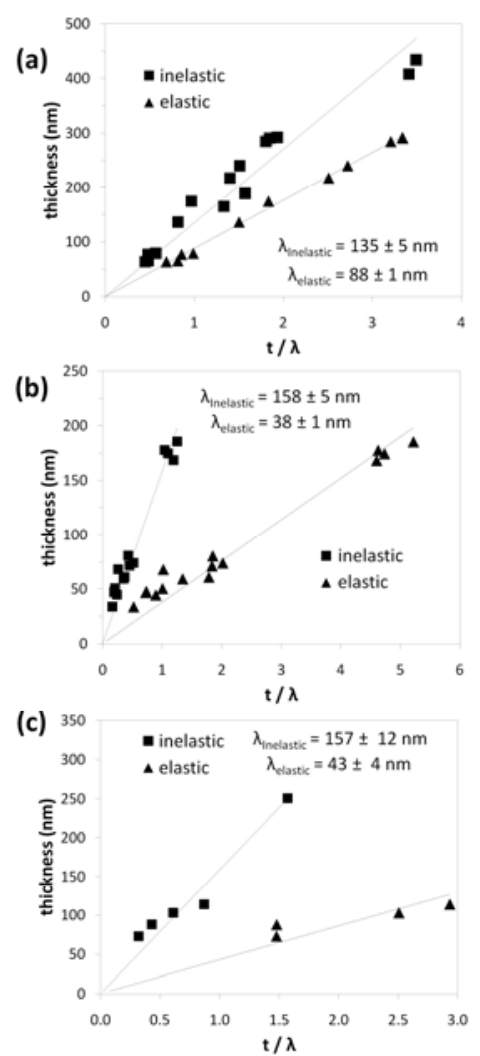

FIG. 3 Absolute thickness vs scattering thickness for (a) AYFC, (b) ZCA, and (c) CZ. The slopes of the fit lines are the EMFP and IMFP. 